\title{
Electronic structure of cylindrical simple-metal nanowires in the stabilized jellium model
}

\author{
N. Zabala* \\ Elektrika eta Elektronika Saila, UPV-EHU 644 P.K., 48080 Bilbo, Spain
}

\author{
M. J. Puska and R. M. Nieminen \\ Laboratory of Physics, Helsinki University of Technology, P.O. Box 1100, FIN-02015 HUT, Finland
}

(Received 15 June 1998)

\begin{abstract}
The ground-state electronic structures of cylindrical quantum wires are studied within the stabilized jellium model and using the spin-dependent density-functional theory. The subband structure is shown to affect the cohesive properties, causing an oscillating structure in the force needed to elongate the wire. Because the steps in the quantized conductance reflect also the subband structure a correlation between the force oscillations and conductance steps is established. The model also predicts magnetic solutions commensurate with the subband structure and consequently additional steps in the conductance. [S0163-1829(99)02719-8]
\end{abstract}

\section{INTRODUCTION}

The jellium model, treated within the density functional theory (DFT), has provided the basic physical understanding of the electronic structures of simple metal surfaces, ${ }^{1}$ vacancies and voids inside metals, ${ }^{2}$ and finite clusters of simple metal atoms. ${ }^{3}$ The scheme simplifies the electron-ion interaction by smearing the ions to a rigid positive background charge of constant density. However, the electron-electron interactions are treated more carefully using, for example, the local-density approximation for electron exchange and correlation effects. ${ }^{4}$ The effect of the actual crystal structure in stabilizing the metal to a certain density can be taken into account by adding a simple correction to the external potential. In the so-called stabilized jellium model ${ }^{5}$ this correction is just a constant in the region of the positive background charge. The stabilized jellium model can often give, besides qualitative understanding and trends, also results of quantitative significance.

In this paper we apply the stabilized jellium model to get insight into the electronic and cohesive properties of extremely thin metallic wires of nanometer dimensions (nanowires). ${ }^{6-8}$ The physical properties of nanowires reflect the quantum-mechanical phenomena due to the confinement of the valence-electron wave functions in the plane perpendicular to the wire axis. For example, the electrical conductance will be quantized. ${ }^{9}$ Recently, it has been realized that the characteristic electronic structure has also a direct influence on the ionic structure of the nanowire, and it is not possible to separate cohesive properties and conductance. ${ }^{10-13}$

Experimentally, metallic nanowires can be produced by several different ways. The simplest scheme is to put two metallic protrusions in contact and then pull them from each other over atomic distances: a nanowire is produced, which upon pulling is elongated and narrowed, until it eventually breaks. This basic mechanism is employed, for example, in the scanning tunneling microscopy (STM) studies of nanowires. ${ }^{14}$ Breaking of macroscopic wires produces also nanowires. The breaking can be done using the sophisticated mechanically controllable break-junctions technique, ${ }^{7}$ but even simpler arrangements are sufficient. ${ }^{15,16}$ An interesting possibility is to produce nanowires by filling carbon or other kinds of nanotubes. ${ }^{17}$ In the experiments the conductance is usually monitored as a function of the elongation of the nanowire. Moreover, in the atomic force microscope (AFM) experiments by Rubio, Agrait, and Vieira, ${ }^{18}$ the conductance and the force during the formation and rupture of $\mathrm{Au}$ contacts have been measured simultaneously. A clear correlation between the force oscillations and the conductance steps during the elongation of the nanowire was seen.

Modeling of the formation of metallic nanowires in a STM experiment was first done by molecular-dynamics simulations in which the atomic structure was solved using many-atom-type interaction potentials. ${ }^{19,20}$ The simulations showed that the elongation takes place through successive stress accumulation and relief stages. The calculated structures were then used to determine electric conductance by counting the available conductive channels. The weakness of this procedure is that the direct correspondence between the cohesive and conduction properties through the valence electron structure is broken. The first-principles moleculardynamics simulations based on solving the self-consistent electron structures remedy this deficiency. ${ }^{8,21}$ Indeed, this kind of simulation ${ }^{8,22}$ shows that the atomic geometries at the neck can be derived from those of isolated small atomic clusters, the stability of which derives from the closed-shell structures of valence electrons. The role of the valenceelectron structure is emphasized in jellium-type models, ${ }^{10-13}$ which completely ignore the detailed ionic structure. In these calculations the confinement of the valence electrons in the direction perpendicular to the wire results in an electronlevel structure, the subbands of which are gradually emptied as the wire elongates or its radius decreases. As a result, cohesive properties, such as the elongation force will show oscillations as the radius of the wire reduces. Moreover, because the subbands constitute the conductance channels, the conductance shows simultaneously a steplike behavior in correlation with the force oscillations.

We calculate electronic properties of cylindrical wires of stabilized jellium. The electron structures are solved selfconsistently using DFT, within the local spin density (LSD) 
approximation for electron exchange and correlation. ${ }^{4}$ The results help us to understand several properties of metallic nanowires and to predict new phenomena. For example, we have previously shown that simple metal quantum wires may have spontaneous magnetization for certain radii. ${ }^{23}$ In this paper we report systematically on the cohesive properties of the stabilized jellium wires. Here our experiment is a direct continuation of that by Yannouleas and Landman. ${ }^{10}$ They solved the electronic structures of Na-jellium wires using the so-called shell-correction method, which uses non-selfconsistent electronic structures from an extended ThomasFermi theory, but takes the single-electron shell structure into account as a correction. Moreover, switching from the jellium to the stabilized jellium model enables us also the study of the trends between different simple metals, i.e., as a function of the average bulk valence-electron density. The next step towards a more realistic description of the actual nanowires is to consider a jellium wire with variable cross section, i.e., a wire with a narrow neck region. As a matter of fact, Yannouleas et $a l^{24}$ have made this step with their computationally efficient shell correction method. One of their main conclusions is that the cohesive and transport properties of the wires are determined to a large extent by the electronic structure at the narrowest part of the wire. This conclusion justifies the use of model with a constant radius as a first approach to the problem.

The rest of the paper is organized as follows. In Sec. II we describe the practical features of the model used to calculate the electronic ground-state configurations. In Sec. III we discuss the results for the electronic structure and cohesive properties. In Sec. IV we study the appearance of magnetic solutions and rationalize the findings by using the Stoner criterium for ferromagnetism. Section V contains the conclusions.

\section{THEORY}

In this section we describe the application of the stabilized jellium model to calculate the electronic structures of quantum wires. We use atomic units throughout the text: The length and energy are given in the units $a_{0}=\hbar / m e^{2}$ and 1 Hartree $=m e^{4} / \hbar^{2}$, respectively. We consider infinitely long jellium cylinders so that the two parameters completely defining the systems are the equilibrium bulk valence-electron density given in terms of the usual $r_{s}$ parameter and the radius $R$ of the cylinder. The rigid positive-background charge density is

$$
n_{+}(r)=\bar{n} \theta(R-r),
$$

where $\bar{n}=3 /\left(4 \pi r_{s}^{3}\right)$ and $\theta(r)$ is the Heaviside step function. The number of electrons per unit length $N_{z}=\bar{n} \pi R^{2}$ neutralizes the positive-background charge so that the electron density has a radial distribution $n_{-}(r)$.

The ground-state electron structure is obtained within the DFT theory by solving self-consistently the Kohn-Sham equations, thereby minimizing the total energy $E\left[n_{-}^{\uparrow}(r), n_{-}^{\downarrow}(r)\right]$ as a functional of the spin densities $n_{-}^{\uparrow}(r)$ and $n_{-}^{\downarrow}(r) .{ }^{4}$ Within the LSD, the equations read as

$$
\begin{gathered}
\left\{-\frac{\nabla^{2}}{2}+V_{e f f}^{\sigma}(r)\right\} \Psi_{i}^{\sigma}(\mathbf{r})=\epsilon_{i}^{\sigma} \Psi_{i}^{\sigma}(\mathbf{r}), \\
n_{-}(r)=\sum_{\sigma} \sum_{i}^{o c c}\left|\Psi_{i}^{\sigma}(\mathbf{r})\right|^{2},
\end{gathered}
$$

and

$$
V_{e f f}^{\sigma}(r)=v_{c}(r)+v_{x c}^{\sigma}\left[n_{-}^{\uparrow}(r), n_{-}^{\downarrow}(r)\right]-\bar{n} \frac{d\left(t_{0}+\epsilon_{x c}\right)}{d \bar{n}} \theta(R-r) .
$$

Above, $\sigma=\uparrow$ or $\downarrow$ for spin-up and spin-down electrons, respectively. The index $i$ stands for orbital quantum numbers, and the summation to calculate the density runs over all occupied states. In the effective potential $V_{e f f}^{\sigma}(r)$ the first term $v_{c}$ on the right-hand side includes the Coulomb interaction with the total electron density $n_{-}(r)=n_{-}^{\uparrow}(r)$ $+n_{-}^{\downarrow}(r)$ and with the positive background, i.e.,

$$
v_{c}(r)=\int \frac{n_{-}\left(r^{\prime}\right)-n_{+}\left(r^{\prime}\right)}{\left|\mathrm{r}-\mathrm{r}^{\prime}\right|} d \mathrm{r}^{\prime} .
$$

The second term $v_{x c}$ gives the exchange-correlation potential within the LSD, for which we use the Perdew-Zunger parametrization ${ }^{25}$ of the exchange-correlation data by Ceperley and Alder. ${ }^{26}$ The last term is the stabilization potential ${ }^{5}$ containing terms due to the bulk kinetic $\left(t_{0}\right)$ and exchangecorrelation $\left(\epsilon_{x c}\right)$ energies per electron.

The Kohn-Sham eigenfunctions $\Psi_{i}^{\sigma}(\mathbf{r})$ for the infinite cylindrical geometry are written as

$$
\Psi_{m n k_{z}}^{\sigma}(r, \phi, z)=\frac{e^{i k_{z} z}}{\sqrt{L}} \frac{e^{i m \phi}}{\sqrt{2 \pi}} R_{m n}^{\sigma}(r),
$$

where $m=0, \pm 1, \pm 2, \ldots$ is the azimuthal quantum number, and $n=1,2,3, \ldots$ is the radial quantum number related to the number of radial nodes $(n-1)$ of the radial wave function $R_{m n}^{\sigma}(r)$. Further, $k_{z}$ is the wave vector associated to the axial $z$ direction along which the electrons have no restriction to move, and $L$ is the normalization length along the $z$ axis. It is useful to do the substitution $U_{m n}^{\sigma}(r)=r^{1 / 2} R_{m n}^{\sigma}(r)$, so that the one-particle Schrödinger equation (2.2) leads to the differential equation

$$
\frac{d^{2} U_{m n}^{\sigma}(r)}{d r^{2}}+\left(2\left[\epsilon_{m n}^{\sigma}-V_{e f f}^{\sigma}(r)\right]-\frac{\left(m^{2}-1 / 4\right)}{r^{2}}\right) U_{m n}^{\sigma}(r)=0 .
$$

This is of the same form as the radial equation studied in spherically symmetric problems. We obtain the radial wave functions $U_{m n}^{\sigma}(r)$ and the eigenvalues $\epsilon_{m n}^{\sigma}$ by solving the differential equation with a point-and-shoot method in a radial point mesh. Different types of methods for the cylindrical symmetry were employed, e.g., by Proetto ${ }^{27}$ and Östling et al. ${ }^{28}$ The eigenenergies,

$$
\epsilon_{m n k_{z}}^{\sigma}=\frac{k_{z}^{2}}{2}+\epsilon_{m n}^{\sigma},
$$


correspond to parabolic subbands with the bottoms at the energies $\epsilon_{m n}^{\sigma}$.

In the infinite cylindrical geometry the spin densities read as

$$
n_{-}^{\sigma}(r)=\frac{1}{2 \pi^{2}} \sum_{\substack{m, n \\ \epsilon_{\sigma}^{m n} \leqslant \epsilon_{F}}}\left|R_{m n}^{\sigma}\right|^{2} \sqrt{2\left(\epsilon_{F}-\epsilon_{m n}^{\sigma}\right)} .
$$

Above, the Fermi energy $\epsilon_{F}$ is obtained self-consistently from the neutrality condition

$$
N_{z}=\frac{1}{\pi} \sum_{\sigma} \sum_{\substack{m, n \\ \epsilon_{m n}^{\sigma} \leqslant \epsilon_{F}}} \sqrt{2\left(\epsilon_{F}-\epsilon_{m n}^{\sigma}\right)} .
$$

The Coulomb potential arising from the total charge density $n_{-}+n_{+}$is calculated using the Green's function ${ }^{29}$ corresponding to Poisson equation in the cylindrical geometry as

$$
\begin{aligned}
v_{c}(r)= & 2 \int_{r}^{\infty} 2 \pi r^{\prime}\left[n_{-}\left(r^{\prime}\right)+n_{+}\left(r^{\prime}\right)\right] \ln \left(\frac{R_{0}}{r^{\prime}}\right) d r^{\prime} \\
& +2 \ln \left(\frac{R_{0}}{r}\right) \int_{0}^{r} 2 \pi r^{\prime}\left[n_{-}\left(r^{\prime}\right)+n_{+}\left(r^{\prime}\right)\right] d r^{\prime} .
\end{aligned}
$$

Above, $R_{0}$ is an arbitrary finite radius. In practice, the Coulomb potential is zero beyond a radius at which the electron density is vanishingly small.

\section{RESULTS}

In this section we present results for stabilized jellium wires corresponding to $\mathrm{Al}, \mathrm{Na}$, and Cs metals. The stabilized jellium correction is small for $\mathrm{Na}$ because the bulk electron gas is stable at a density close to $r_{s}=3.93$, which we use for $\mathrm{Na}$. The correction is essential for $\mathrm{Al}\left(r_{s}=2.07 \mathrm{a}_{0}\right)$ and $\mathrm{Cs}$ $\left(r_{s}=5.62 \mathrm{a}_{0}\right)$, which in the plain jellium model would expand or shrink in volume, respectively. The stabilized jellium will give for these metals physically meaningful surface energies and elongation forces without losing the simplicity of the uniform background model.

\section{A. Electronic ground-state configuration}

Figure 1 shows the exchange-correlation, Coulomb, and total effective potentials as a function of the distance from the cylinder axis for a $\mathrm{Na}$ wire having the radius $R=7.8 \mathrm{a}_{0}$. The ground state of this wire is spontaneously spin polarized and the effective potentials for the spin-up and spin-down electrons differ slightly from each other. The potential well is mainly due to the exchange-correlation contribution. In the electrostatic potential the surface-dipole barrier is about 1 $\mathrm{eV}$, i.e., of the same order as the barrier for a planar surface. ${ }^{30}$ The bottom energies of the first $(|m|, n)$ subbands below the Fermi level and the Fermi level itself are also given. The two lowest subbands are split as a result of the spin polarization. Figure 2 gives the total electron density $n_{-}^{\uparrow}(r)+n_{-}^{\downarrow}(r)$ and the spin density $n_{-}^{\uparrow}(r)-n_{-}^{\downarrow}(r)$ corresponding to the wire in Fig. 1. The spin moment per unit length for this system is about $0.1 \mu_{B}$ per conduction elec-

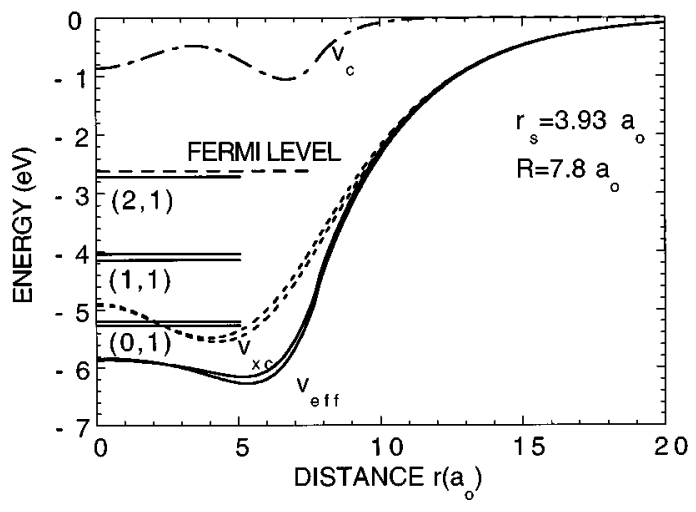

FIG. 1. Na stabilized-jellium wire $\left(r_{s}=3.93 \mathrm{a}_{0}\right.$ and $\left.R=7.8 \mathrm{a}_{0}\right)$. The Coulomb potential $v_{c}$ (dash-dotted line), the exchangecorrelation potential $v_{x c}$ (dotted line), and the effective potential (solid line) are given as a function of the distance from the cylinder axis. The bottoms of the filled-energy subbands labeled $(|m|, n)$ (solid lines) and the Fermi level (dashed line) are shown as well.

tron, i.e., per $\mathrm{Na}$ atom. The spin density and spin moment derive mainly from the highest subband, which is totally spin polarized. The contributions from the lower spin-splitted subbands are small (see also Fig. 6, below). The electron density has its maximum around the middle of the axis and the edge of the positive-background charge. The electron density spills out from the region of positive charge a few atomic units. The spin density has its maximum value away from the cylinder axis near the jellium edge. This tendency would be even stronger for spin polarized wires of larger radii, because in them the polarization results from subbands with higher $|m|$ values.

The energy eigenvalues obtained for narrow $\mathrm{Na}$ wires are considered in Fig. 3, giving the bottoms of the filled subbands and the positions of the Fermi level as a function of the cylinder radius $R$. As the wire becomes wider, new subbands dive below the Fermi level and attain occupancy. The subbands are filled in the sequence $(|m|, n)=(0,1),(1,1)$, $(2,1),(0,2),(3,1) \ldots$, giving for the degeneracy ratios the sequence of $1,2,2,1,2, \ldots$. This sequence was first predicted by Bogachek et al. ${ }^{32}$ and it has been seen in the conductance steps measured for Na nanowires. ${ }^{7}$ The Fermi-level

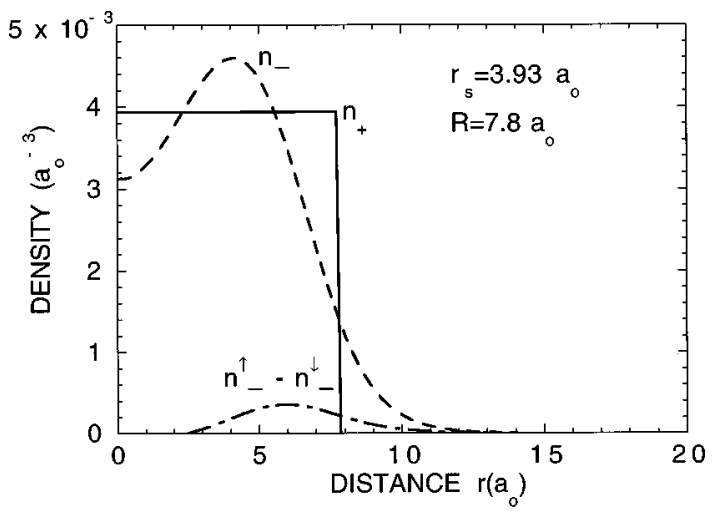

FIG. 2. Na stabilized-jellium wire $\left(r_{s}=3.93 \mathrm{a}_{0}\right.$ and $\left.R=7.8 \mathrm{a}_{0}\right)$. The total electron density $n_{-}^{\uparrow}(r)+n_{-}^{\downarrow}(r)$ (dashed line), the spin density $n_{-}^{\uparrow}(r)-n_{-}^{\downarrow}(r)$ (dash-dotted line), and the positive background charge $n_{+}$(solid line) are given as a function of the distance from the cylinder axis. 


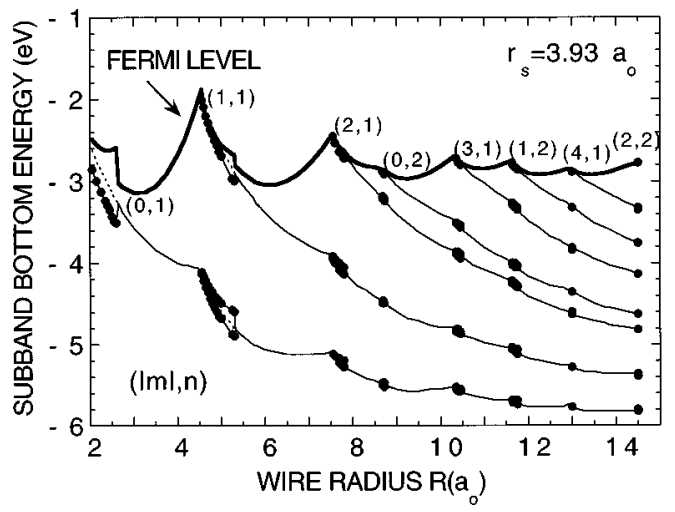

FIG. 3. Energy eigenvalues of Na stabilized-jellium wires $\left(r_{s}\right.$ $=3.93 \mathrm{a}_{0}$ ). The bottoms of different subbands (thin solid lines) and the Fermi level (thick solid line) are given as a function of the wire radius. The eigenvalues corresponding to spin-polarized solutions (black circles) as well as to unstable spin-compensated solutions (dashed lines) are shown separately.

position shows oscillations so that a peak appears each time a new subband starts to be populated. Another feature, shown in the figure with black dots, is the existence of spinpolarized configurations. They appear in intervals associated with the openings of new subbands. For small radii, the splitting of the subbands occupied by both spin species and the lowering of the bottom of the totally spin-polarized (highest occupied) subband with respect to the spin-compensated calculation, marked with a dashed line, are clearly seen. When the spin-compensated state is recovered the Fermi level jumps downwards.

The electron work function, i.e., the distance between the Fermi level and vacuum, can be directly read from Fig. 3 for cylindrical stabilized jellium wires with different radii, because the energy zero coincides with the vacuum level (see Fig. 1). The work function oscillates due to the subband structure and has sharp minima at the opening of a new subband and discontinuities at transitions between spincompensated and spin-polarized states. As the radius $R$ increases the work function approaches that for a planar surface. Apart from the effects due to spin-polarized solutions the present work function results agree well with those obtained by Yannouleas and Landman ${ }^{10}$ with the shell correction method. For Na the stabilized-jellium correction affects only slightly the electronic structure.

\section{B. Surface energy and elongation force}

The surface energy of a cylindrical stabilized-jellium wire can be determined from the total energy per unit length $E\left[n_{-}^{\uparrow}, n_{-}^{\downarrow}\right] / L$ as

$$
\sigma=\frac{1}{2 \pi R}\left\{E_{\text {tot }}\left[n_{-}^{\uparrow}, n_{-}^{\downarrow}\right] / L-\pi R^{2} \bar{n}\left[\frac{3}{5} \epsilon_{F}(\bar{n})+\epsilon_{x c}(\bar{n})\right]\right\} .
$$

Above, $\epsilon_{F}(\bar{n})$ is the Fermi energy of the homogeneous electron gas measured with respect to the bottom of the energy bands. The surface energy is plotted in Fig. 4 as a function of the wire radius $R$ for $\mathrm{Al}, \mathrm{Na}$, and $\mathrm{Cs}$ nanowires. The curves for the different metals are rather similar when the radii $R$ are scaled with $r_{s}$. The surface energies exhibit oscillations with
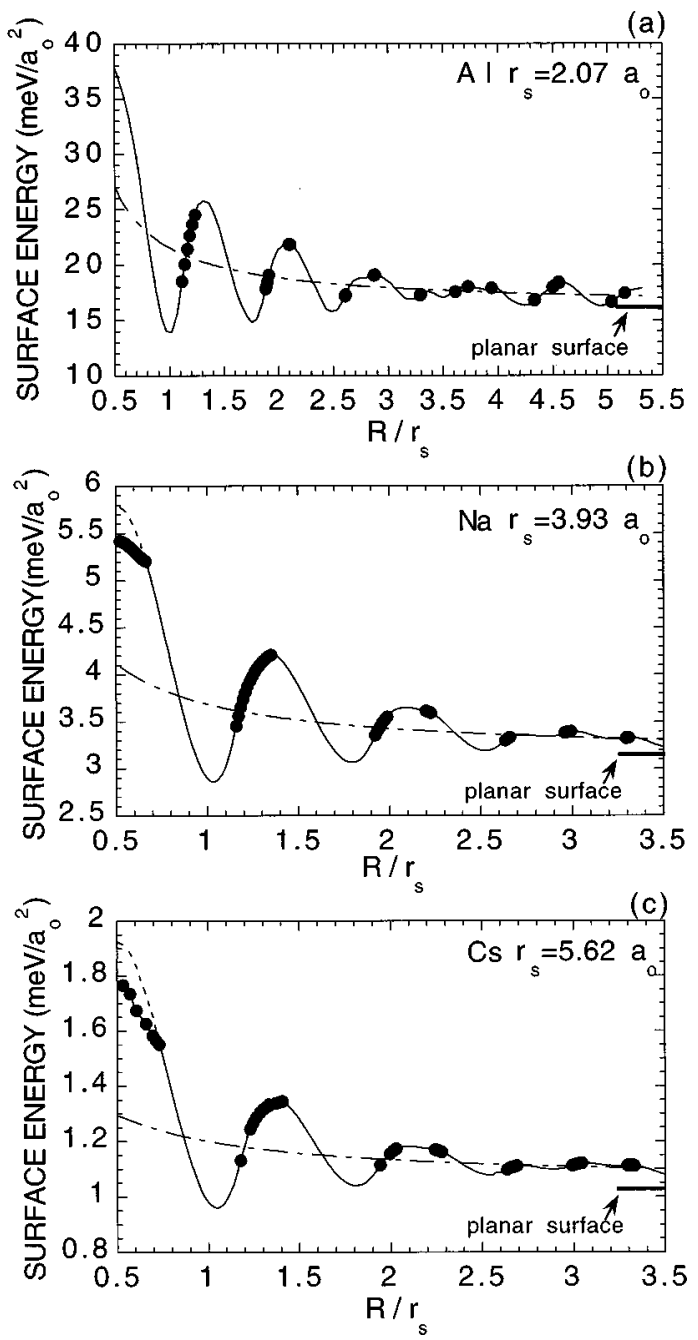

FIG. 4. Surface energy for Al (a), Na (b), and Cs (c) stabilizedjellium wires as a function of the wire radius (solid lines). The values corresponding to spin-polarized solutions (black circles) as well as to unstable spin-compensated solutions (dashed lines) are shown separately. The results of the extended liquid-drop model are also given (dash-dotted lines). When the wire radii increase the surface energies approach those (Ref. 31) for planar stabilizedjellium surfaces (thick horizontal lines).

minima slightly before the occurrence of new subbands and maxima at a small finite occupancy of the highest subband. The spin polarization, when stable, lowers slightly the surface energy. When the wire radius increases the surface energies approach the values of the planar surfaces, ${ }^{31}$ which are $16.15 \mathrm{meV} a_{0}^{-2}$ for $\mathrm{Al}, 3.14 \mathrm{meV} a_{0}^{-2}$ for $\mathrm{Na}$, and 1.055 $\mathrm{meV} a_{0}^{-2}$ for Cs. We want to underline at this point the importance of using the stabilized jellium model: the plain jellium model would predict negative values for the Al surface energy.

The dash-dotted lines in Fig. 4 correspond to an extended liquid-drop model ${ }^{31}$ in which the surface energy of a cylindrical wire is

$$
\sigma(R)=\sigma_{\text {planar }}+\frac{\gamma}{4 R}+\frac{\delta}{4 \pi(2 R)^{2}}+O\left(1 / R^{3}\right) .
$$

Above, $\sigma_{\text {planar }}$ is the surface energy for a planar surface, $\gamma$ is the curvature energy taking into account the breaking 
(forming) of lateral bonds on a convex (concave) surface, and $\delta$ is an additional fitting parameter. All three parameters have been determined in Ref. 31 using the stabilized jellium model for voids in bulk $\mathrm{Al}, \mathrm{Na}$, and $\mathrm{Cs}$. In order to obtain Eq. (3.2) from the corresponding equation for a void one has to change the sign of the curvature (energy) and substitute the curvature of a void, i.e., the inverse of the void radius, by $(1 / R+1 / \infty) / 2=1 /(2 R)$. Here $R$ and $\infty$ are the two principal curvature radii of the cylindrical surface. We see that the extended liquid-drop model gives nicely the descending trend, undressed from the oscillations due to the subband structure. Moreover, the model shows quantitatively how slowly the cylinder surface energies approach those of planar surfaces.

In order to discuss the stability of the jellium wires we consider deformations that conserve the volume of a long wire with length $L$ and radius $R$. The wires corresponding to minima in the total energy per unit volume are then (meta) stable and their radii play a similar role as the magic numbers discussed in the context of small atomic clusters. ${ }^{3}$ Wires with slightly larger (smaller) radii would in this model without any external support spontaneously lengthen (shorten). The corresponding force is called the elongation force because, when negative, it will oppose the increase of the length of the wire. In terms of the surface energy, the elongation force is given as a function of the wire radius as

$$
F=-\frac{d E}{d L}=-\frac{d(2 \pi R L \sigma)}{d L}=-\pi R \sigma+\pi R^{2} \frac{d \sigma}{d R} .
$$

The first term on the right-hand side is due to the increase of the surface area when the wire elongates. The second term is nonzero because of the dependence of the surface energy on the wire radius. It has components due to the change of the curvature (energy) and due to the energy oscillations reflecting the subband structure.

The elongation force is shown in Fig. 5 for $\mathrm{Al}, \mathrm{Na}$, and $\mathrm{Cs}$ wires. In the case of $\mathrm{Na}$ wires [Fig. 5(b)] we have given its decomposition into two components corresponding to the two terms on the right-hand side of Eq. (3.3). The first contribution, due to the increase of the surface area, has a slightly descending and moderately oscillating behavior. It follows quite closely the force $-\pi R \sigma_{\text {planar }}$ (the straightsolid line), derived by using the planar surface energy $\sigma_{\text {planar }}$. In order to obtain this physically correct trend for all metals it is important to use the stabilized jellium model instead of the plain jellium model. The second contribution to the total force, shown with the dash-dotted line, contains the prominent oscillations of the amplitude, being of the order of $0.8 \mathrm{nN}$. The oscillation amplitude scales approximately as $\epsilon_{F}(\bar{n}) / \lambda_{F}(\bar{n})$, where $\lambda_{F}(\bar{n})$ is the Fermi wavelength. ${ }^{11}$ This results in a cubic dependence on the inverse of $r_{s}, \epsilon_{F}(\bar{n}) / \lambda_{F}(\bar{n})=9 / 16 r_{s}^{3}$, giving for $\mathrm{Al}, \mathrm{Na}$, and Cs force oscillations of about $5.22,0.76$, and $0.26 \mathrm{nN}$, respectively. The behavior of the total force for $\mathrm{Na}$ agrees well with the results by the shell-corrected method. ${ }^{10}$

The AFM experiments show stress accumulation and relief stages as the nanowire elongates. The measured minima (maxima in the tensile stress) for gold samples ${ }^{18}$ corresponding to the narrowest wires have a similar magnitude and an ascending trend as the wire lengthens as our prediction for
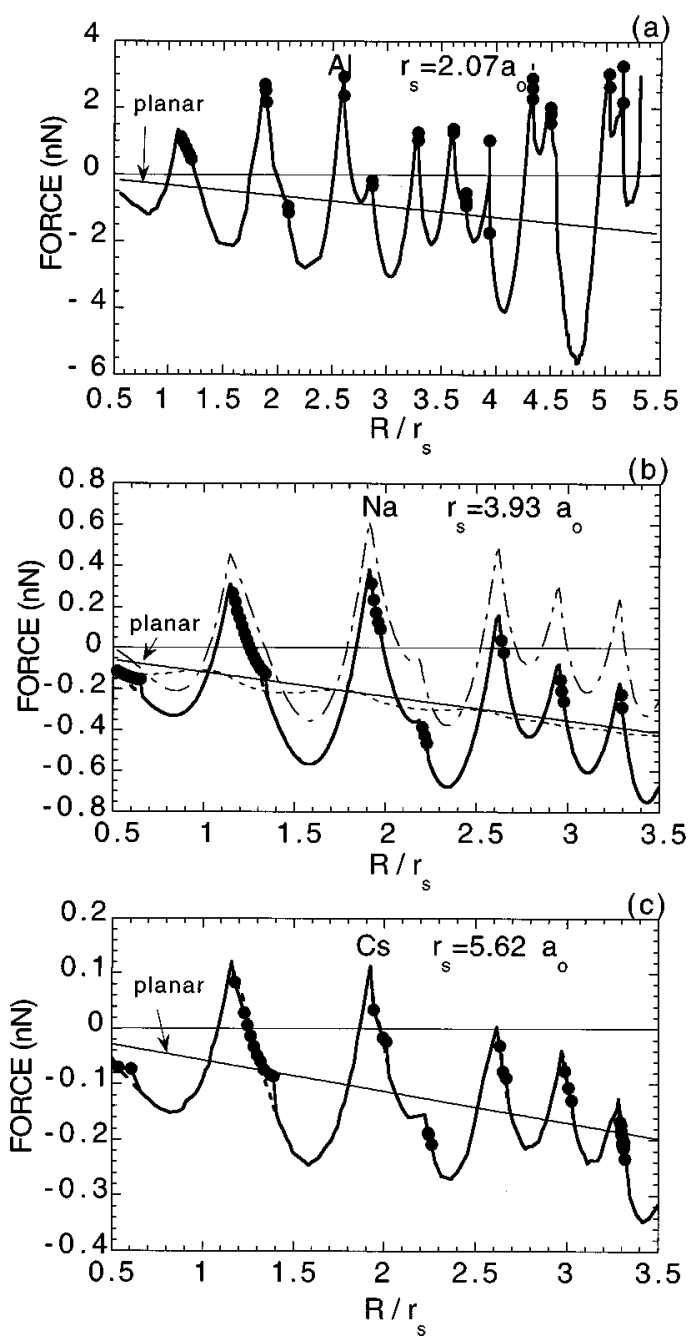

FIG. 5. Elongation force for $\mathrm{Al}$ (a), $\mathrm{Na}$ (b), and $\mathrm{Cs}$ (c) stabilized-jellium wires as a function of the wire radius (thick solid lines). The values corresponding to spin-polarized solutions (black circles) as well as to unstable spin-compensated solutions (dashed lines) are shown separately. The forces derived from the surface energy of a planar surface $\left(-\pi R \sigma_{\text {planar }}\right)$ are shown for comparison (narrow solid lines). For $\mathrm{Na}$ wires, the contributions $-\pi R \sigma$ (dotted line) and $\pi R^{2}(d \sigma / d R)$ (dash-dotted line) are given.

the $\mathrm{Al}\left(r_{s}=2.07 \mathrm{a}_{0}\right)$ wire. In our calculations, the energy minima correspond to the zeroes in the force associated with positive slopes in Fig. 5. The decrease of the force from these points as the wire radius decreases would mean the accumution of stress as the wire is elongated. After the following force minimum there would be a relief stage in which the magnitude of the force needed for elongation first decreases and then the wire would elongate spontaneously even without pulling. The experiments do not show the positive parts of the force, but this kind of behavior has been seen also by Yannouleas and Landman ${ }^{10}$ in a jellium calculation, which included similar energy terms as our calculation. As a function of the elongation, the energy minima occur just after a subband has been emptied. Then during the stress accumulation stage the conductivity is predicted to be constant, in accordance with the AFM experiment. ${ }^{18}$ The energy relief stage associated with an emptying of the subband and a steplike decrease in the conductance seems to be much longer in 
our model calculations than in the actual experiment. The correlation of the force oscillations and the quantum structure of the conductance has been predicted in many jelliumtype calculations. ${ }^{10-13}$

When comparing the theoretical and experimental results one should bear in mind that the stabilized jellium model predicts cohesive properties merely qualitatively, due to the smearing of the actual ionic cores. For example, one can try to describe a linear atomic chain by a narrow stabilized jellium cylinder. In the case of a Na-atom chain only the first subband of the jellium wire should have occupancy. The radius of the jellium wire corresponding to the energy minimum is then, according to Fig. 5(b), $\sim 4.2 \mathrm{a}_{0}$, which, by considering the positive charge per unit length, corresponds to the interatomic distance of $\sim 4.6 \mathrm{a}_{0}$. This is an unreasonably short distance when comparing, e.g., with interatomic distances in bulk Na metal. Similarly, in the case of an Alatom chain the second subband (corresponding to valence $p$ electrons) should be the highest occupied subband. The energy minimum at $R \approx 3.6 \mathrm{a}_{0}$ gives the short interatomic distance of about $2.8 \mathrm{a}_{0}$. In spite of this deficiency one should, however, recognize that the stabilized jellium model describes well the electronic properties of simple metal systems if one constrains the shape of the background charge to correspond to the atomistic system in question. ${ }^{33}$

\section{Magnetic solutions, Stoner criterion}

As discussed above in the context of energy eigenvalues (Fig. 3), the cylindrical stabilized jellium wires have magnetic ground-state solutions. They appear so that the highestenergy subband is totally spin polarized when its occupancy is small enough. The spin polarization lowers the total energy and it affects the surface energy and the elongation force as is indicated in Figs. 4 and 5 by the regions of black circles.

For small cylinder radii only the first subband is occupied and for the smallest radii it is eventually spin polarized. This kind of spontaneous spin polarization has been predicted also by Gold and Calmels ${ }^{34}$ for cylindrical wires using the exchange-correlation energy of a quasi-one-dimensional electron gas. When more than one subband is occupied, the highest subband, when totally polarized, induces a magnetic moment in the lower subbands, too. However, this induction is weak so that the magnetic moment per electron decreases when the number of occupied subbands increases. This is shown in Fig. 6 giving the magnetic moment per electron in Bohr magnetons as a function of the radius for a $\mathrm{Na}$ wire. The moment decreases in order to recover the nonmagnetic character of the simple bulk metal for large cylinder radii. One can also notice that when a new subband starts to be occupied, the magnetic moment increases nearly linearly until a maximum value, from which it falls abruptly to zero. In the inset of Fig. 6 the maximum magnetic moment due to the totally polarized second subband is given as a function of the $r_{s}$ parameter, i.e., corresponding to the different simple metals. The contributions of the first $(0,1)$, and the second $(1,1)$, subbands are also plotted, showing that the main contribution comes clearly from the upper subband. If this maximum spin moment is calculated per unit length it varies surprisingly weakly, namely, for $2<r_{s}<6 \mathrm{a}_{0}$ it is nearly constant, 0.11

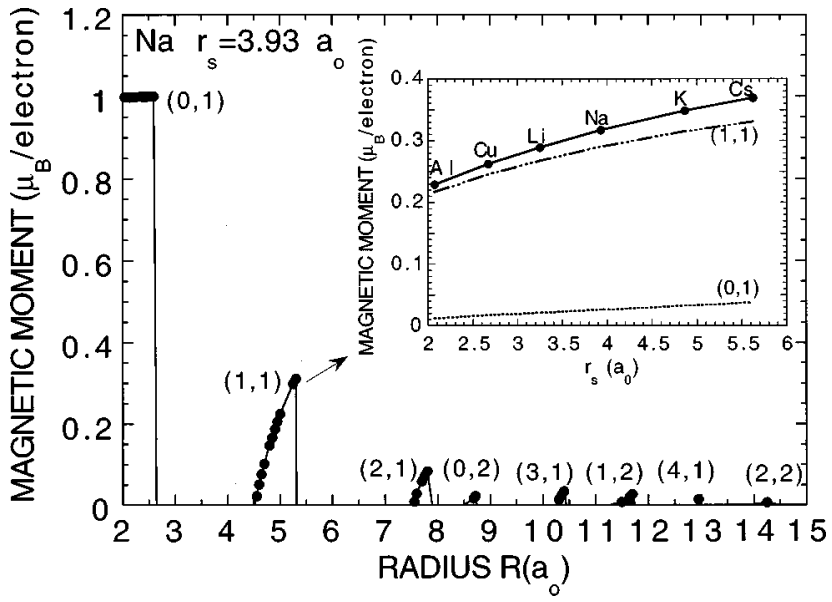

FIG. 6. Magnetic moment per electron (in Bohr magnetons) for Na stabilized-jellium wires $\left(r_{s}=3.93 \mathrm{a}_{0}\right)$ as a function of the wire radius. The inset shows the maximum magnetic moment per electron when the second subband is occupied as a function of the electron-density parameter $r_{s}$. The contributions due to the $(0,1)$ (dotted line) and $(1,1)$ (dash-dotted line) subbands are distinguished.

$\pm 0.01 \mu_{\mathrm{B}} / \mathrm{a}_{0}$. The insensitivity is due to a cancellation effect: The radius corresponding to the maximum moment increases roughly linearly with $r_{s}$, decreasing the moment per unit length, but the spin polarization per electron, as shown in the inset of Fig. 6, increases with increasing $r_{s}$.

We can analyze the appearance of magnetic ground-state solutions of simple metal nanowires by making use of the Stoner criterion of ferromagnetism in bulk metals. The occurrence of magnetic solutions in the beginning of the occupancy of each new subband would suggest the application of Hund's rules for finite systems. These rules have recently been applied to account for the magnetic solutions in quantum dots. ${ }^{35}$ But the number of electron states in a subband is not limited in our case because a jellium wire is infinite in the $z$ direction. From the viewpoint of an infinite system it is natural to ask if the appearance of the magnetism in the jellium wires could resemble that in bulk metallic systems, i.e., if the Stoner criterion is applicable. According to this criterion, ferromagnetism exists whenever the condition

$$
I \widetilde{D}\left(E_{F}\right)>1,
$$

is fulfilled. Above, $\widetilde{D}\left(E_{F}\right)$ is the density of states (DOS) per atom in a spin-compensated system at the Fermi level and $I$ is the Stoner parameter, an "exchange" integral, which includes also the electron-electron correlation effects.

The Stoner parameter I should, in principle, be calculated using the wave functions of the system at the Fermi level. ${ }^{36,37}$ However, for simplicity we use the result valid for the homogeneous electron gas and define $I$ per electron,

$$
I=\frac{8\left[\epsilon_{x c}^{F}(\bar{n})-\epsilon_{x c}^{P}(\bar{n})\right]}{9\left(2^{4 / 3}-2\right)} .
$$

Above, $\epsilon_{x c}^{F}\left(\epsilon_{x c}^{P}\right)$ is the exchange-correlation energy per electron in a totally spin-polarized (spin-compensated) electron gas. For simple metals this equation gives nearly the same results as full band-structure calculations. As a matter of fact, 


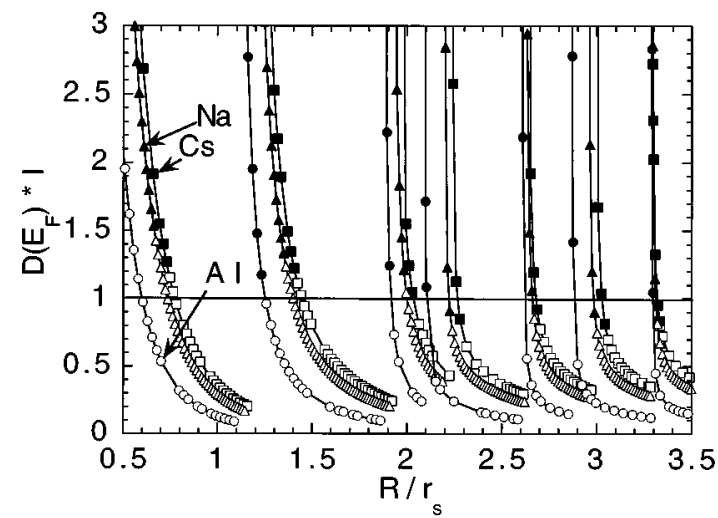

FIG. 7. Stoner criterion product $I \widetilde{D}$ for $\mathrm{Al}$ (circles), $\mathrm{Na}$ (triangles), and Cs (squares) stabilized-jellium wires as a function of the wire radius. The regions of stable magnetic and spincompensated solutions are denoted by filled and open markers, respectively.

in them the dependence of $I$ on the parametrization of the exchange-correlation energy is stronger than the effect of the jellium approximation. For example, the Perdew-Zunger parametrization $^{25}$ of the exchange-correlation data by Ceperley and Alder, ${ }^{26}$ which we use in this paper gives for $\mathrm{Na}$ the value $I=0.72 \mathrm{eV}$, whereas in the Gunnarsson-Lundqvist ${ }^{38}$ parametrization $I=0.94 \mathrm{eV}$, i.e., close to the band-structure value of $0.91 \mathrm{eV}$ calculated with this parametrization by Janak. $^{36}$

The DOS per unit volume is calculated for the infinitecylinder geometry as

$$
\operatorname{DOS}(E)=\frac{1}{\pi R^{2}} \sum_{\sigma} \sum_{m, n} \frac{1}{\pi \sqrt{2}} \frac{1}{\sqrt{E-\epsilon_{m, n}^{\sigma}}} .
$$

Thus, it is a superposition of the DOS's corresponding to different one-dimensional subbands. The factor $1 / \pi R^{2}$ in the front changes the DOS per unit length to the DOS per unit volume. The DOS exhibits a strong peak whenever the energy is close to the bottom of a subband. With the above $I$, defined for the homogeneous electron gas, one has to use for $\widetilde{D}\left(E_{F}\right)$ the DOS per electron. It is obtained from Eq. (3.6) by multiplying by the volume per electron $\left[\left(4 \pi r_{s}^{3}\right) / 3\right]$ and by taking the spin degeneracy into account,

$$
\widetilde{D}\left(E_{F}\right)=\frac{8 r_{s}^{3}}{3 \sqrt{2} \pi R^{2}} \sum_{m, n} \frac{1}{\sqrt{E_{F}-\epsilon_{m, n}}} .
$$

This equation shows that a high $r_{s}$ value (low-electron density) as well as a small wire radius $R$ increase $\widetilde{D}\left(E_{F}\right)$ and favor the occurrence of ferromagnetism.

The product $I \widetilde{D}\left(E_{F}\right)$ is plotted in Fig. 7 as a function of the wire radius $R$ for stabilized jellium wires corresponding to $\mathrm{Al}, \mathrm{Na}$, and $\mathrm{Cs}$. The filled markers mean systems for which we have found in self-consistent electronic-structure calculations stable magnetic solutions, i.e., the total energy of a spin-polarized solution is lower than that of a spincompensated one. The open markers denote then stable, spin-compensated systems. The correlation between the fulfillment of the Stoner criterion and the existence of magnetic solutions is almost quantitative, especially when the moment is due to the polarization of the second or a higher subband. For the Al wire the Stoner criterion with the above approximations would predict that the total polarization of the first subband survives to much larger radii than it does in actual calculations. In accordance with Eq. (3.7) the peaks become narrower when the radius $R$ increases and the probability of finding magnetic solutions decreases. One can also see the trend that the magnetic solutions reach to relatively larger radii [to smaller values of the $I \widetilde{D}\left(E_{F}\right)$ product] when $r_{s}$ increases.

The above form of the Stoner criterion is valid for a system at zero temperature. The model gives also an estimate for the critical temperature ${ }^{39}$

$$
T_{c}=I \mu / 4 k_{B},
$$

where $\mu$ is the magnetic moment per electron in Bohr magnetons and $k_{B}$ is the Boltzmann constant. For a $\mathrm{Na}$ wire ( $I$ $=0.72 \mathrm{eV}$ ) with the second subband polarized to the maximum moment of about $0.3 \mu_{B}$, this gives $620 \mathrm{~K}$ for the critical temperature. This can be considered as an upper-limit order-of-magnitude estimate, because the above equation is known to overestimate critical temperatures for bulk solids. Anyway, the result predicts that magnetic moments of simple metal nanowires could survive to high finite temperatures. As the wire radius increases, the decrease of the magnetic moment per atom would lower $T_{c}$.

Thus far we have considered the appearance and stability of the magnetic solutions by assuming that the ionic structure or, in our model, the given shape of the jellium background, is stable. Figure 5 shows that the magnetic solutions are associated with positive values of the elongation force, or at least they appear during the stress-relief stages if the nanowire is being pulled. This would speak against the observation of magnetic states in AFM experiments. On the other hand, as discussed above, the stabilized jellium model describes the cohesive properties only qualitatively. Atomistic first-principles simulations are needed to clarify the stability of the magnetic solutions with respect to changes in the ionic structure. $^{33}$

During the elongation of a nanowire the appearance of a magnetic moment would mean a quantum step of $e^{2} / h$ in the conductance. The observation of conductance steps of this size might be hindered by a simultaneous orbital degeneracy of the highest occupied subband. The degeneracy actually favors the magnetic solution. However, it is encouraging that experimental evidence of this kind for magnetic "fingerprints" exists in the conductance measurements of quantumpoint contacts at the interface between two different bulk semiconductors. ${ }^{40}$ Moreover, for the two-dimensional electron gas in a ribbonlike geometry the calculations by Wang and Berggren ${ }^{41}$ predict that systems with one or more subbands may be magnetic, provided that the electron density of the highest-occupied subband is low enough. Our finding of spontaneous magnetic moments in simple metal nanowires is also related to the prediction of Weber et $a l^{42}$ that a vanadium atom chain will be ferromagnetic for any number of atoms in it. A second consequence of the appearance of a magnetic moment would be that during the pulling a discontinuity appears in the elongation force. This is seen in Fig. 5 most clearly when the occupancy of the first or second sub- 
band of $\mathrm{Na}$ or $\mathrm{Cs}$ wires is low. The discontinuity results from different slopes of the total energy in the spin-polarized and spin-compensated solutions. ${ }^{23}$

In the present model we have assumed perfect translation symmetry along the wire. A more realistic model would include constrictions at which the wire radius locally decreases (examples of that kind of models include Refs. 11) and 24). For a constriction geometry we expect our prediction of magnetic solutions also to be valid in the sense that the local DOS at the Fermi level and at the constriction will for certain radii be so high that the Stoner criterion is fulfilled.

\section{CONCLUSIONS}

We have calculated electronic properties of infinitely long stabilized-jellium cylinders. The model, in contrast with the plain-jellium model, is capable to mimic physically reasonably all simple metals, irrespective of their average valenceelectron density. Thus, we have considered the whole range of electron densities by simulating $\mathrm{Al}, \mathrm{Na}$, and $\mathrm{Cs}$ nanowires. For $\mathrm{Na}$ our results agree well with those obtained by Yannouleas and Landman ${ }^{10}$ in the plain jellium using the shellcorrection method. The prominent feature of the electronic structures calculated are the subbands. They are due to the confinement perpendicular to the cylinder axis and influence the cohesive properties of the wires: There exist radii of enhanced stability corresponding to the total-energy minima and the elongation force oscillates as a function of the wire radius. The subband structure also affects other electronic properties, such as the quantization of the conductance and the oscillations of the electron work function. Thereby the stabilized-jellium model can qualitatively account for the correlation between the conductance steps and the force oscillations seen in the AFM experiments.

Our calculations predict the existence of stable magnetic solutions for certain critical-wire radii, at which the occupancy of the highest subband below the Fermi level is small. The existence of magnetism has been explained on the basis of the Stoner criterion for ferromagnetism of bulk metals. We have also discussed the stability of the magnetic solutions and their possible detection in experiments.

\section{ACKNOWLEDGMENTS}

We are grateful to U. Landman, K.-F. Berggren, M. Manninen, and N. Nestle for useful communications. N. Z. acknowledges the financial support of the Spanish Ministry of Education (DGES), Project Reference PR95-591, and the Laboratory of Physics of the HUT for hospitality.
*Also at Materialen Fisika Saila, UPV-EHU 1072 P.K., 20080 Donostia, Spain.

${ }^{1}$ N. D. Lang, in Theory of the Inhomogeneous Electron Gas, edited by S. Lundqvist and N. H. March (Plenum, New York, 1983), p. 309.

${ }^{2}$ M. Manninen, R. M. Nieminen, P. Hautojärvi, and J. Arponen, Phys. Rev. B 12, 4012 (1975).

${ }^{3}$ W. A. de Heer, Rev. Mod. Phys. 65, 611 (1993); M. Brack, ibid. 65, 677 (1993).

${ }^{4}$ R. O. Jones and O. Gunnarsson, Rev. Mod. Phys. 61, 689 (1989).

${ }^{5}$ J. P. Perdew, H. Q. Tran, and E. D. Smith, Phys. Rev. B 42, 11627 (1990); H. B. Shore and J. H. Rose, Phys. Rev. Lett. 66, 2519 (1991).

${ }^{6}$ Nanowires, Vol. 340 of NATO Advanced Studies Institute, Series E: Applied Sciences, edited by P. A. Serena and N. Garcia (Kluwer, Dordrecht, 1997).

${ }^{7}$ J. M. Krans, J. M. van Ruitenbeek, V. V. Fisun, I. K. Yanson, and L. J. Jongh, Nature (London) 375, 767 (1995).

${ }^{8}$ R. N. Barnett and U. Landman, Nature (London) 387, 788 (1997).

${ }^{9}$ R. Landauer, J. Phys.: Condens. Matter 1, 8099 (1989); C. W. J. Beenakker and H. van Houten, in Solid State Physics, edited by H. Ehrenreich and D. Turnbull (Academic, New York, 1991), Vol. 44, p. 1.

${ }^{10}$ C. Yannouleas and U. Landman, J. Phys. Chem. B 101, 5780 (1997).

${ }^{11}$ C. A. Stafford, D. Baeriswyl, and J. Bürki, Phys. Rev. Lett. 79, 2863 (1997).

${ }^{12}$ J. M. van Ruitenbeek, M. H. Devoret, D. Esteve, and C. Urbina, Phys. Rev. B 56, 12566 (1997).

${ }^{13}$ S. Blom, H. Olin, J. L. Costa-Krämer, N. Garcia, M. Jonson, P. A. Serena, and R. I. Shekhter, Phys. Rev. B 57, 8830 (1998).

${ }^{14}$ J. I. Pascual, J. Mendez, J. Gomez-Herrero, A. M. Baro, N. Garcia, U. Landman, W. D. Luedtke, E. N. Bogachek, and H. P.
Cheng, Science 267, 1793 (1995).

${ }^{15}$ J. L. Costa-Krämer, N. Garcia, P. Garcia-Mochales, and P. A. Serena, Surf. Sci. Lett. 342, L1144 (1995).

${ }^{16}$ N. Garcia and J. L. Costa-Krämer, Europhys. News 89, 27 (1996).

${ }^{17}$ C. Guerret-Piecourt, Y. Le Bouar, A. Loiseau, and H. Pascard, Nature (London) 372, 761 (1994); A. Rubio, J. L. Corkill, and M. L. Cohen, Phys. Rev. B 49, 5081 (1994).

${ }^{18}$ G. Rubio, N. Agraï, and S. Vieira, Phys. Rev. Lett. 76, 2302 (1996).

${ }^{19}$ U. Landman, W. D. Luedtke, N. Burnham, and R. J. Colton, Science 248, 454 (1990).

${ }^{20}$ M. Brandbyge et al., Phys. Rev. B 52, 8499 (1995); M. Brandbyge, K. W. Jacobsen, and J. K. Nørskov, ibid. 55, 2637 (1997); M. R. Sørensen, M. Brandbyge, and K. W. Jacobsen, ibid. 57, 3283 (1998).

${ }^{21}$ Jian Wang, Hong Guo, J. L. Mozos, C. C. Wan, G. Taraschi, and Qingrong Zheng, Phys. Rev. Lett. 80, 4277 (1998).

${ }^{22}$ H. Häkkinen and M. Manninen, Europhys. Lett. 44, 80 (1998).

${ }^{23}$ N. Zabala, M. J. Puska, and R. M. Nieminen, Phys. Rev. Lett. 80, 3336 (1998).

${ }^{24}$ C. Yannouleas, E. N. Bogachek, and U. Landman, Phys. Rev. B 57, 4875 (1998).

${ }^{25}$ J. P. Perdew and A. Zunger, Phys. Rev. B 23, 5048 (1981).

${ }^{26}$ D. M. Ceperley and B. J. Adler, Phys. Rev. Lett. 45, 566 (1980).

${ }^{27}$ C. R. Proetto, Phys. Rev. B 45, 11911 (1992).

${ }^{28}$ D. Östling, D. Tománek, and A. Rosén, Phys. Rev. B 55, 13980 (1997).

${ }^{29}$ J. D. Jackson, Classical Electrodynamics (Wiley, New York, 1975), pp. 115-118.

${ }^{30}$ N. D. Lang and W. Kohn, Phys. Rev. B 1, 4555 (1970).

${ }^{31}$ P. Ziesche, M. J. Puska, T. Korhonen, and R. M. Nieminen, J. Phys.: Condens. Matter 5, 9049 (1993). 
${ }^{32}$ E. N. Bogachek, A. N. Zagoskin, and I. O. Kulik, Fiz. Nizk. Temp. 16, 1404 (1990) [Sov. J. Low Temp. Phys. 16, 796 (1990)].

${ }^{33}$ The ability of the stabilized-jellium model to describe the electronic structures of atomic chains has been studied by $\mathrm{A}$. Ayuela, M. J. Puska, and R. M. Nieminen (unpublished).

${ }^{34}$ A. Gold and L. Calmels, Philos. Mag. Lett. 74, 137 (1996); L. Calmels and A. Gold, Solid State Commun. 106, 139 (1998).

${ }^{35}$ M. Koskinen, M. Manninen, and S. M. Reimann, Phys. Rev. Lett. 79, 1389 (1997).

${ }^{36}$ J. F. Janak, Phys. Rev. B 16, 255 (1977).

${ }^{37}$ O. Gunnarsson, J. Phys. F 6, 587 (1976).

${ }^{38}$ O. Gunnarsson and B. I. Lundqvist, Phys. Rev. B 13, 4274 (1976).
${ }^{39}$ H. Ibach and H. Lüth, Solid-State Physics (Springer, Berlin, 1991).

${ }^{40}$ K. J. Thomas, J. T. Nicholls, M. Y. Simmons, M. Pepper, D. R. Mace, and D. A. Ritchie, Phys. Rev. Lett. 77, 135 (1996); R. D. Tscheuschner and A. D. Wieck, Superlattices Microstruct. 20, 615 (1996); P. Ramvall, N. Carlsson, I. Maximov, P. Omling, L. Samuelson, W. Seitert, Q. Wang, and S. Lourdudoss, Appl. Phys. Lett. 71, 918 (1997).

${ }^{41}$ C.-K. Wang and K.-F. Berggren, Phys. Rev. B 54, R14 257 (1996).

${ }^{42}$ S. E. Weber, B. K. Rao, P. Jena, V. S. Stepanyuk, W. Herger, K. Wildberger, R. Zeller, and P. H. Dederichs, J. Phys.: Condens. Matter 9, 10739 (1997), and references therein. 